Reprod. Nutr. Dévelop., 1980, 20 (1 B), 203-207.

\title{
Fonction thyroïdienne et métabolisme de l'iode chez l'embryon de Poulet
}

\author{
par Nicole DAUGERAS-BERNARD, F. LACHIVER \\ avec la collaboration technique de Françoise BOULU-LAPOINTE \\ Laboratoire de Physiologie générale ef comparée du Muséum national d'Histoire naturelle \\ ef Laborafoire d'Endocrinologie comparée ossocié au C.N.R.S., 7, rue Cuvier, 75005 Paris.
}

Summary. Thyroid function and iodine metabolism in the chicken embryo.

lodine is accumulated in the egg yolk during oogenesis. It is transierred during incubation through the walls of the yolk sac (extraembryonic endomesoderm surrounding the yolk) to the extraembryonic, and thence to the embryonic circulation. lodine exchanges would probably occur between both compartments, the egg yolk and the embryonic blood in which the iodine level increases from day 10 to 20 of incubation. The increase of thyroid gland iodine content parallels that of the plasma iodine level. Our results suggested that the pituitary gland, which acts directly on the developing thyroid gland, might also influence the iodine exchanges across the yolk sac wall, thereby affecting the thyroid by increasing the iodine supply.

\section{Introduction.}

Lorsque nous avons entrepris l'étude de la fonction thyroïdienne de l'embryon de Poulet, l'essentiel des résultats déjà établis l'avait été en utilisant le radioiode et ne permettait qu'une approche qualitative de cette fonction (références dans Daugeras et Lachiver, 1972).

Dans un premier temps, nous avons analysé le métabolisme intrathyroïdien de l'iode ${ }^{127}$. Cet iode provient essentiellement du jaune ou vitellus de l'œuf où il s'est accumulé au cours de l'ovogenèse. Il s'y trouve surtout (95 p. 100) sous forme d'iodure, $\Gamma$, $(50 \mu \mathrm{g}$ en moyenne, avec une concentration de $290 \mu \mathrm{g} / 100 \mathrm{~g})$. Il est transféré, après passage à travers la paroi de la vésicule vitelline (annexe embryonnaire endo-mésoblastique entourant le vitellus) dans la circulation extra-embryonnaire, puis embryonnaire du poulet et de là, à la glande thyroïde (fig. 1 et 2). C'est ce transfert de l'iodure du jaune vers l'embryon, au cours de l'incubation, que dans un deuxième temps, nous avons étudié. 


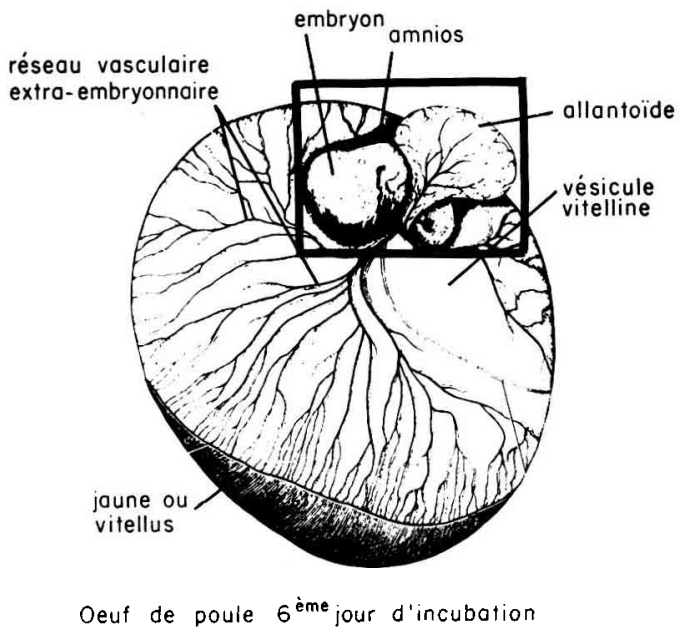

FIG. 1. - Embryon de poulet et annexes embryonnaires au $6^{\mathrm{e}}$ jour d'incubation de l'œuf de poule (d'après Huettner, 1965). La région encadrée est schématisée dans la figure 2.

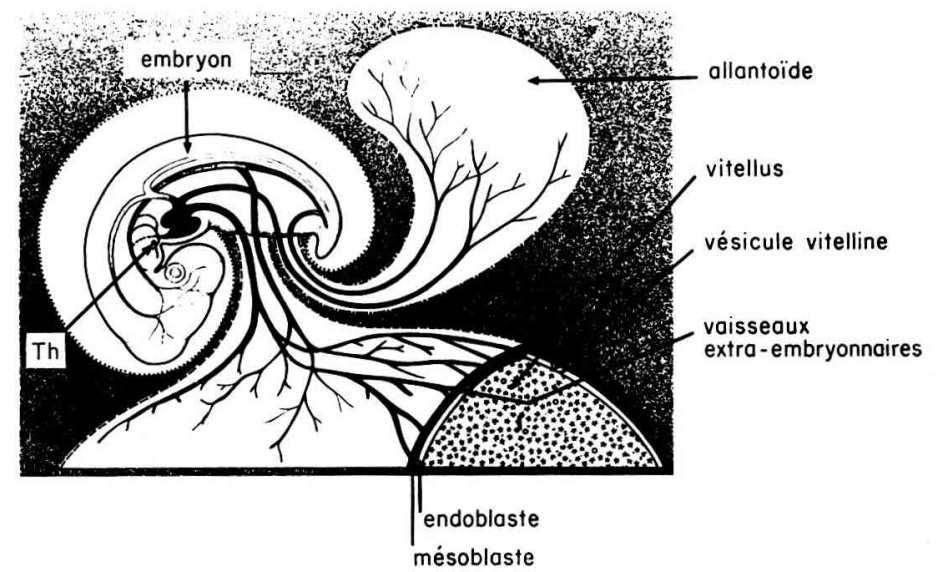

FIG. 2. - Schéma des relations embryon-vésicule vifelline. L'iode du vitellus est transféré à travers l'endoblaste de la vésicule vifelline dans les vaisseaux sanguins extra-embryonnaires différenciés dans le mésoblaste de la vésicule et qui sont en continuité avec le réseau vasculaire de l'embryon.

\section{Matériel et méthodes.}

Les œufs de poule Leghorn blanche sont mis à incuber à $37,8 \pm 0,3{ }^{\circ} \mathrm{C}$.

Les techniques utilisées dans l'étude du métabolisme iodé intrathyroïdien du $8 \mathrm{e}$ au 20 jour d'incubation (détermination des quantités d'iode et de thyroglobuline ( ${ }^{1}$ ) dans la glande, répartition de l'iode entre ses différentes formes, iodure ef iode orga-

(1) Thyroglobuline : protéine support de l'hormonogenèse thyroïdienne. 
nique, répartition dans la thyroglobuline des différents acides aminés iodés ( $\left.{ }^{1}\right)$ ) ont été précédemment décrites (Daugeras ef al., 1976).

Le transfert de l'iodure du vitellus vers l'embryon a été abordé en mesurant quotidiennement la teneur en iodure plasmatique (ou iodurémie) des vaisseaux extra-embryonnaires (fig. 2), du 8 e au 20 e jour d'incubation.

L'éfude des facteurs intervenant dans le métabolisme iodé de l'embryon (métabolisme intrathyroïdien et transfert de l'iode vitellin à l'embryon) a été appréhendée à l'aide de techniques d'hypophysectomie, d'injection d'un ion compétitif de l'iodure, l'anion perchlorique $\mathrm{ClO}_{4}^{-}$, et d'injection d'un autre antithyroïdien, la thiourée, au mécanisme d'action différent de celui du perchlorate.

- L'hypophysectomie est pratiquée par décapitation au $2^{\mathbf{e}}$ jour d'incubation selon la technique classique de Fugo (1940).

- L'injection de thiourée est décrite dans Daugeras (1971).

- $200 \mu \mathrm{l}$ de la solution de perchlorate de Na sont injectés, comme pour la thiourée, au $5^{e}$ jour d'incubation. Elle a une molarité de $0,115 \mathrm{M}$, molarité choisie d'après TixierVidal (1958).

\section{Résultats et discussion.}

L'iode, ${ }^{127}$, est présent dans la thyroïde de l'embryon dès le $7 \mathrm{e}$ jour d'incubation. Sa quantiłé y croît à partir du $10 \mathrm{e}$ jour ef jusqu'à la fin de l'incubation (Daugeras et Lachiver, 1972). Parallèlement, la quantité de thyroglobuline, et son taux d'iodation croissent du $10^{\mathrm{e}}$ au $20^{\mathrm{e}}$ jour. Au cours du développement embryonnaire, l'iode thyroïdien est donc incorporé de plus en plus à la thyroglobuline (TG) dont le pourcentage en acides aminés iodés reste constant du $9 \mathrm{e}$ au $20^{\mathrm{e}}$ jour d'incubation (12 p. 100 de l'iode de la TG est de l'iode thyroxinien $\left(T_{4}\right)$ (Daugeras et al., 1976).

L'iodurémie évolue parallèlement à l'iode thyroidien. Au 8 e jour, l'iodure est présent dans la circulation embryonnaire, mais à très faible concentration, et c'est seulement à partir du 10 e jour que cette concentration croît pour atteindre $80 \mu \mathrm{g} \mathrm{I} \times \mathrm{dl}^{-1}$ au 20e jour (Lachiver ef al., 1973).

Il y a donc augmentation, au cours de l'incubation, de la quantité d'iodure vitellin fourni à l'embryon par passage à travers la paroi de la vésicule vitelline. Mais, conjointement à ce transfert d'iodure du vitellus au sang embryonnaire, il existerait un passage d'iodure dans le sens embryon-vitellus. Il est suggéré par les travaux de Wollman et Zwilling (1953) ef par ceux d'Okonski, Lengemann ef Comar (1960) montrant que l'iode radioactif injecté à l'embryon au $7 \mathrm{e}$ ou au $14 \mathrm{e}$ jour est transféré très rapidement dans le vifellus.

Il existerait donc, à travers la paroi de la vésicule vitelline, dès les $7 e_{-} 8$ e jours, des échanges permanents bidirectionnels d'iodure entre les deux compartiments vitellus et sang embryonnaire.

D'une part, la concentration en I- du vitellus supérieure à celle du plasma de l'embryon, d'autre part le sens du gradient électrique, l'embryon étant électro-

(1) Acides aminés iodés : MIT, 3-monoiodotyrosine ; DIT, 3,5-diiodotyrosine ; $T_{3}, 3,5,3^{\prime}$-triiodothyronine; $T_{4}$, thyroxine. 
positif par rapport au vitellus (calculé à partir des concentrations ioniques en $\mathrm{Na}^{+}, \mathrm{K}^{+}, \mathrm{Cl}^{-}$, du jaune de l'œuf et du plasma; Romanoff, 1967 ; Lehoux, 1974) nous ont conduits à émettre l'hypothèse suivante : le transport d'iodure du vitellus vers l'embryon serait passif, le transport inverse contre le gradient électrochimique serait actif (fig. 3).

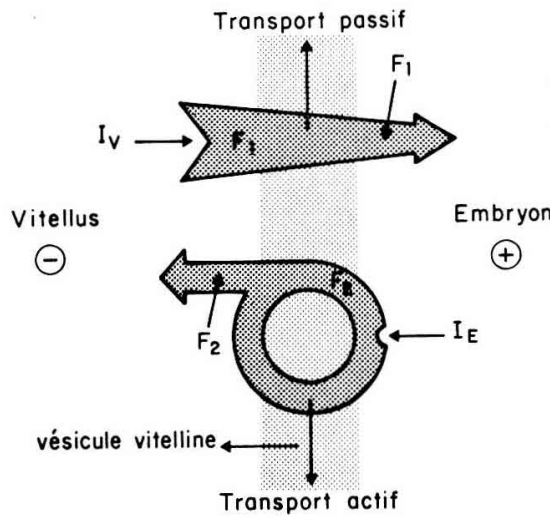

FIG. 3. - Modèle proposé des échanges d'iode à travers la paroi de la vésicule vitelline. $I_{V}=$ lodure vifellin ; $I_{E}=$ lodure en provenance du compartiment embryonnaire $; F_{1}=$ Transport passif de l'iodure du vitellus vers l'embryon ; $F_{2}=$ Transport actif de l'iodure de l'embryon vers le vitellus.

C'est pour tenter de vérifier ce modèle d'échanges à travers la paroi de la vésicule vitelline, que nous avons étudié les conséquences sur la concentration en iodure du plasma embryonnaire, de l'injection dans le vitellus d'un ion compétitif de $\mathrm{I}^{-}$dans son transport actif, l'anion perchlorique $\mathrm{ClO}_{4}^{-}$.

L'injection dans le vitellus du perchlorate entraîne, dès le 8 e jour, une augmentation de l'iodurémie qui persiste jusqu'à la fin de l'incubation : 8 e jour - Témoins : $1,3 \mu \mathrm{gl}^{-} \times \mathrm{dl}^{-1} ;$ - Injectés : $49 \mu \mathrm{g} \mathrm{I}^{-} \times \mathrm{dl}^{-1} ; 18 \mathrm{e}$ jour - Témoins : $37 \mu \mathrm{I}^{-} \times \mathrm{dl}^{-1}$; - Injectés : $160 \mu \mathrm{g} \mathrm{I}^{-} \times \mathrm{dl}^{-1}$. Ces données sont tout à fait compatibles avec le modèle proposé pour les échanges d'iodure vitellus $\rightleftarrows$ plasma (fig. 3 ). Le perchlorate injecté dans le vitellus ne s'oppose pas au transport " passif» de l'iodure dans la circulation embryonnaire : une diminution de l'iode fixé par la thyroïde des embryons traités témoigne de la présence dans leur circulation de cet antithyroïdien. Puis, il entre en compétition avec l'iodure dans son transport « actif» embryon $\rightarrow$ vitellus, entraînant ainsi une augmentation de l'iodure présent dans la circulation embryonnaire.

Les évolutions synchrones, à partir du $10^{\mathrm{e}}$ jour, des paramètres thyroïdiens étudiés (Daugeras ef Lachiver, 1972 ; Daugeras ef al., 1976), et du flux net d'iodure, $F_{1}-F_{2}$ (fig. 3) (Lachiver ef al., 1973), suggèrent une action hypophysaire aux deux niveaux, thyroïdien et paroi de la vésicule vitelline, car c'est au 10e jour que s'établit l'axe hypophyso-thyroïdien de l'embryon de poulet (Tixier-Vidal, 1958). De fait, l'hypophysectomie de l'embryon entraîne à partir du $11 \mathrm{e}$ jour à la fois une diminution de l'iodurémie et un arrêt de fixation de l'iode par la thyroïde (Lachiver, Daugeras et Boulu, 1976). L'action de l'hypophyse au niveau de la paroi de la vésicule vitelline pourrait être soit directe, soit indirecte par l'intermédiaire des hormones thyroïdiennes, leur sécrétion 
éłant très fortement diminuée au $11^{\mathrm{e}}$ jour, après hypophysectomie (Daugeras-Bernard, Leloup et Lachiver, 1976).

L'injection dans la vésicule vitelline d'un antithyroïdien, la thiourée, bloquant la thyroïde, entraîne la quasi-disparition des hormones thyroïdiennes plasmatiques. La concentration en iodure du plasma embryonnaire des embryons traités est identique à celle des témoins jusqu'au $10^{\mathrm{e}}$ jour. A partir du $11 \mathrm{e}$ jour, elle est supérieure à celle des témoins. Donc, la quantité d'iodure transférée du vitellus à l'embryon est, à partir du $11 \mathrm{e}$ jour, augmentée chez les embryons à sécrétion thyroïdienne bloquée, alors qu'elle est diminuée chez les embryons privés d'hypophyse (Lachiver, Daugeras et Boulu, 1976). Ces résultats suggèrent donc une action directe de l'hypophyse au niveau de la paroi de la vésicule vitelline, l'augmentation de l'iodurémie des embryons soumis aux antithyroïdiens étanł vraisemblablement due à une hypersécrétion de TSH consécutive à la diminution du taux des hormones thyroïdiennes.

\section{Conclusion.}

L'hypophyse de l'embryon de poulet, vraisemblablement par action de la TSH, agirait à partir des $10^{\mathrm{e}}-11^{\mathrm{e}}$ jours d'incubation sur les échanges d'iodure vitellus $\rightleftarrows$ embryon effectués à travers la paroi de la vésicule vitelline (échanges déjà mis en place aux $7 \mathrm{7}_{-} 8^{\mathrm{e}}$ jours). Elle interviendrait donc sur le métabolisme iodé thyroïdien en agissant aussi sur l'apport d'iode à la glande au cours de l'incubation.

\section{5e Réunion du groupe Développement I.N.R.A., Clermont-Ferrand/Theix, 17-18 mai 1979.}

\section{Références}

DAUGERAS N., 1971. Influence de la thiourée sur la charge en glycogène du foie de l'embryon de poulet. J. Embryol. exp. Morphol., 25, 377-384.

DAUGERAS N., BRISSON A., LAPOINTE-BOULU F., LACHIVER F., 1976. Thyroidal iodine metabolism during the development of the chick embryo. Endocrinology, 98, 1321-1331.

DAUGERAS N., LACHIVER F., 1972. Evolution de l'iode, ${ }^{127}$, total thyroïdien chez l'embryon de poulet au cours de l'incubation. J. Embryol. exp. Morph., 27, 615-622.

DAUGERAS-BERNARD N., LELOUP J., LACHIVER F., 1976. Evolution de la thyroxinémie au cours du développement de l'embryon de poulet. Influence de l'hypophysectomie. C. R. Acad. Sci. Paris, Sér. D, 283, 1325-1327.

FUGO N. W., 1940. Effects of hypophysectomy in the chick embryo. J. exp. Zool., 85, 271-297.

HUETTNER A. F., 1965. Fondamentals of comparative embryology of the vertebrates. Mac Millan, New York.

LACHIVER F., DAUGERAS N., BOULU F., 1976. Contrôle hypophysaire du transport de l'iode à travers la paroi du sac vitellin de l'embryon de poulat. J. Physiol. Paris, 72, 99A (abstr.).

LACHIVER F., DAUGERAS N., BRISSON A., BOULU F., 1973. Métabolisme de l'iode ${ }^{127}$ ] de l'embryon de poulet au cours de l'incubation. $5^{\mathrm{e}}$ Conf. Assoc. eur. sur la thyroide, Jérusalem (abstract 62).

LEHOUX J. G., 1974. Aldosterone biosynthesis and presence of cytochrome P-450 in the adrenocortical tissue of the chick embryo. Molec. cell. Endocr., 2, 43-58.

OKONSKI J., LENGEMANN F. W., COMAR C. L., 1960. The utilization of egg iodine by the chicken embryo. J. exp. Biol., 145, 263-269.

ROMANOFF A. L., 1967. Biochemisiry of the avian embryo. John Wiley and Sons, New York.

TIXIER-VIDAL A., 1958. Etude histophysiologique des relations hypophyse-thyroïde chez l'embryon de poulet. Arch. Anat. microsc. Morph., 47, 235-340.

WOLLMAN S. H., ZWILLING E., 1953. Radioiodine metabolism in the chick embryo. Endocrinology, 52, 526-535. 